\title{
Synthesis of Fluorescein Aldehydes for the Sensitive Detection of L-Cysteine ${ }^{\dagger}$
}

\author{
Jörg Ettenauer ${ }^{1, *}$, Vladislav Semak ${ }^{2}$ and Martin Brandl ${ }^{1}$ \\ 1 Department for Integrated Sensor Systems, Center for Water and Environmental Sensors, Danube \\ University Krems, 3500 Krems, Austria; Martin.Brandl@donau-uni.ac.at \\ 2 Department for Health Sciences and Biomedicine, Center for Biomedical Technology, Danube University \\ Krems, 3500 Krems, Austria; Vladislav.Semak@donau-uni.ac.at \\ * Correspondence: Joerg.Ettenauer@donau-uni.ac.at; Tel.: +43-(0)-2732-893-2792 \\ † Presented at the Eurosensors 2018 Conference, Graz, Austria, 9-12 September 2018.
}

Published: 27 November 2018

\begin{abstract}
Amino acids containing thiol-groups such as Cysteine (Cys), Homocysteine (Hcy), and Glutathione play an important role in a great variety of biological processes. However, too low, but also too high concentrations can have negative effects on human health. Therefore, it is of great importance to sensitively detect these risk factors for cardiovascular and neuronal diseases. Furthermore, Cys is an essential amino acid for the growth of pathogenic water-borne bacteria, like Legionella sp. Hence, a reliable, sensitive and selective Cys-detection method that is incorporated into automated biosensors would represent a great tool for a broad range of biological and medical applications. We describe the simple synthesis of fluorescein aldehyde probes for the detection of biologically important thiols, focusing on Cys. The probes displayed highly sensitive responses to L-Cysteine hydrochloride monohydrate in the range of their physiologically relevant levels in the visible and UV spectral region.
\end{abstract}

Keywords: fluorescein aldehydes; L-Cysteine; detection

\section{Introduction}

Elevated levels of amino acids Cys and Hcy have shown to be associated with an increased risk of myocardial infarction, stroke, and venous thromboembolism. In the case of Cys, neurotoxic effects have been demonstrated in vitro and in vivo [1,2]. However, a lack of Cys can induce several health disorders such as slowed growth, muscle and fat loss, hair depigmentation, and liver or skin damage. Therefore, our goal was the development of a simple and sensitive detection method for Cys to be used in the field of bio-medical applications [3].

\section{Materials and Methods}

\subsection{Chemical Synthesis}

Targeted compounds (1) Fluorescein Monoaldehyde (MOAF) and (2) Fluorescein Dialdehyde (DIAF) were synthesized by a Reimer-Tieman formylation reaction of fluorescein (Figure 1) [4,5]. Briefly, sodium hydroxide ( $125 \mathrm{mM}, 16.0$ equiv) was slowly added to a fine suspension of fluorescein (7.52 mM, CAS: 2321-07-5) in methanol. After complete dissolution of fluorescein, chloroform (37.5 $\mathrm{mM}, 15$ equiv) and $15-C r o w n-5(0.3760 \mathrm{mM}, 0.1$ equiv) were slowly added with aid of syringe. The resulting dark brown clear solution was vigorously stirred at $55{ }^{\circ} \mathrm{C}$ in an oil bath for $42 \mathrm{~h}$. After cooling, the reaction mixture was poured on stirred ice cold $1 \mathrm{M} \mathrm{H}_{2} \mathrm{SO}_{4}$ with aid of minimal amount of distilled water. After acidification ( $\mathrm{pH} 1-2)$ the crude product quickly precipitated. The solid was 
filtered off and washed with ice cold water. The wet filtration cake was frozen and lyophilized to afford dark yellow-brown powder. The crude product was purified by flash column chromatography on silica ( $0 \%$ to $50 \%$ EtOAc in petroleum ether) to remove major part of unreacted fluorescein. The mixture of DIAF and MOAF aldehydes was re-purified by a second flash column chromatography on silica ( $0 \%$ to $50 \%$ EtOAc in $\mathrm{CH}_{2} \mathrm{Cl}_{2}$ ) to yield DIAF (white-beige solid, $20 \mathrm{mg}, 0.7 \%$ ) and MOAF (yellow solid, $94 \mathrm{mg}$, 3.5\%). Synthesized aldehydes were clearly identified by FTIR analysis (4000$650 \mathrm{~cm}^{-1}$, Perkin Elmer FT-IR Spectrometer Spectrum Two equipped with Attenuated Total Reflection (ATR) mode using ZnSe as the optical material) [2]. Furthermore, on TLC analysis, aldehydes DIAF and MOAF gave active spots under UV $(365 \mathrm{~nm}), \mathrm{KMnO}_{4}$ and 2,4-DNP (2,4-dinitrophenylhydrazine hydrochloric acid solution in ethanol) stains. Compared to the synthesized aldehydes, fluorescein had a lower $R_{f}$ and was not 2,4-DNP active.

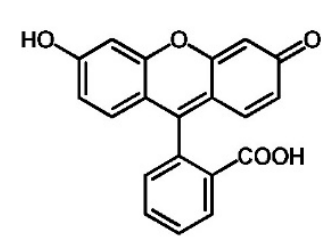

CAS: 2321-07-5

Chemical Formula: $\mathrm{C}_{20} \mathrm{H}_{12} \mathrm{O}_{5}$ Molecular Weight: 332,31

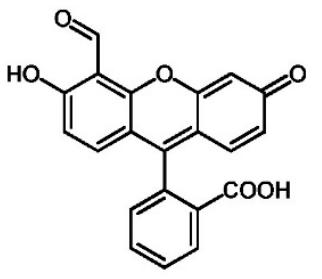

MOAF

Chemical Formula: $\mathrm{C}_{21} \mathrm{H}_{12} \mathrm{O}_{6}$ Molecular Weight: 360,32

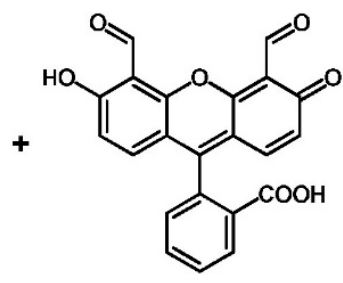

DIAF

Chemical Formula: $\mathrm{C}_{22} \mathrm{H}_{12} \mathrm{O}_{7}$ Molecular Weight: 388,33

Figure 1. Reimer-Tieman formylation of fluorescein.

We were able to separate desired aldehydes from the crude reaction product by flash column chromatography on silica. Synthesized aldehydes were clearly identified by FTIR analysis. Furthermore, DIAF and MOAF TLC spots (comparing to fluorescein, control spot) were active towards 2,4-DNP TLC stain; proof reagent of aldehydes and ketones.

\subsection{Cys Measurements}

Two different methods were used for determination of various L-Cysteine hydrochloride monohydrate (CAS: 7048-04-6) and L-Cys (CAS 52-90-4) concentrations. Therefore, a dilution series of Cys in water was prepared. First, visual analysis of the behavior of mixtures of MOAF $(1 \mathrm{mM})$ or DIAF (0.5 mM), respectively, together with Cys $(0.5$ and $5 \mathrm{mM})$ under visible and UV light (254 and $365 \mathrm{~nm}$ ) using a Vilber Lourmat VL-6.LC UV lamp was done (Figure 2). Secondly, fluorescence measurements with a Perkin Elmer LS55 Luminescence Spectrometer were performed with mixtures of Cys concentrations ranging from $1 \mu \mathrm{M}$ to $1 \mathrm{mM}$ with $2.5 \mu \mathrm{M}$ DIAF. The solutions were excited at $460 \mathrm{~nm}$ and emission spectra were measured from 470 to $650 \mathrm{~nm}$ (Figure 3).

\section{Results and Discussion}

We find that upon addition of L-Cysteine (CAS: 7048-04-6; 0.5-5 mM) to a solution of MOAF (1 $\mathrm{mM}$ ) and DIAF $(0.5 \mathrm{mM})$, a color change from bright yellow to colorless was observed (Figure 2c,f). Higher Cys concentrations ( $5 \mathrm{mM}$ ) led to immediate color reactions whereas for lower concentrations $(0.5 \mathrm{mM})$ several minutes of incubation were necessary. Additionally, fluorescence quenching of MOAF and DIAF solutions with Cys were detected (Figure 2a,b,d,e). DIAF showed to be more sensitive for the L-Cysteine hydrochloride monohydrate detection then MOAF. Fluorescence quenching and color bleaching happened immediately for both tested concentrations (Figure 2e,f). In future experiments a wider concentration range of Cys samples will be analyzed to further evaluate the optical detection limits of this technique. 


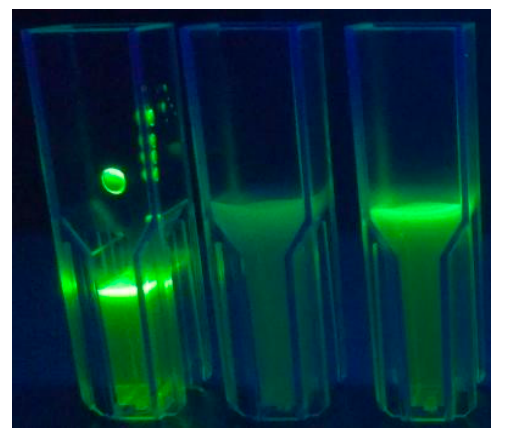

(a)

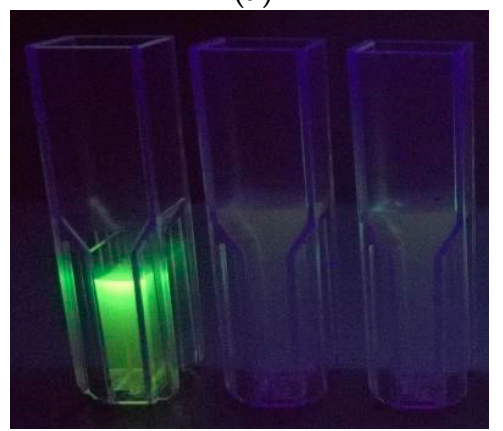

(d)

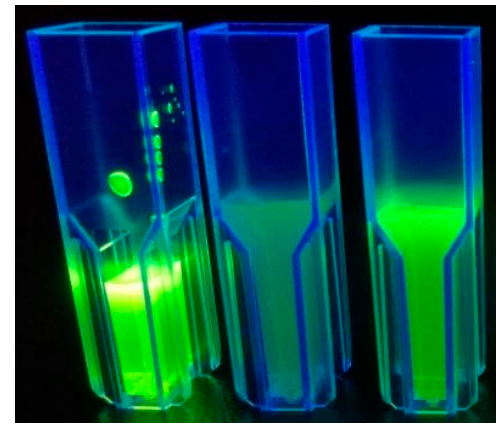

(b)

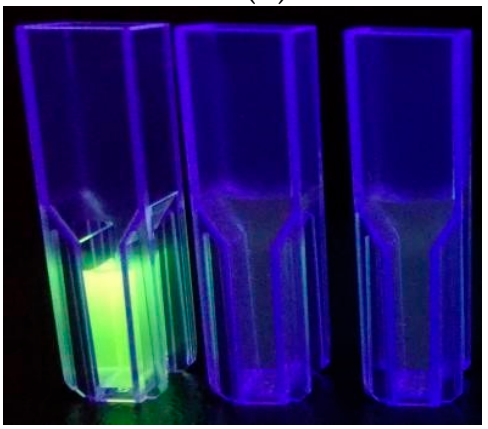

(e)

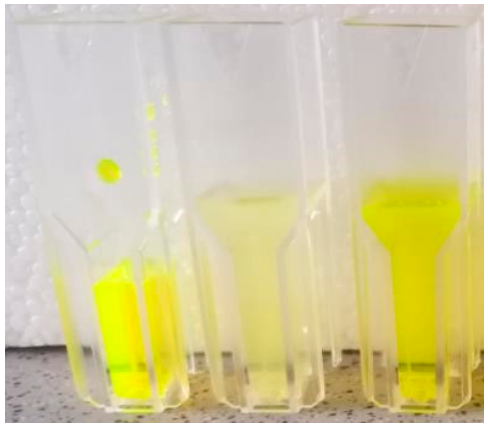

(c)

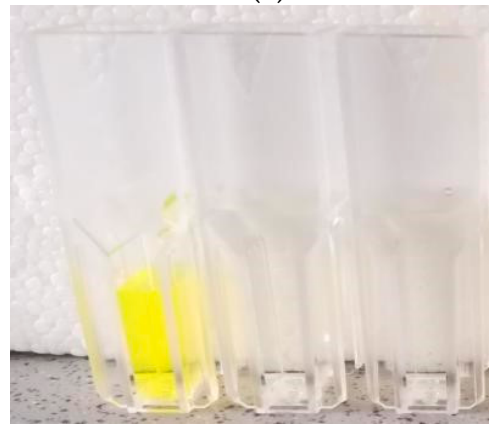

(f)

Figure 2. Color changes of solutions of $\operatorname{MOAF}(\mathbf{a}-\mathbf{c})$ and DIAF $(\mathbf{d}-\mathbf{f})$ with L-Cysteine under UV-light at $254 \mathrm{~nm}(\mathbf{a}, \mathbf{d}), 365 \mathrm{~nm}(\mathbf{b}, \mathbf{e})$ and visible light $(\mathbf{c}, \mathbf{f})$. The left cuvette shows sole MOAF $(1 \mathrm{mM}) / \mathrm{DIAF}$ $(0.5 \mathrm{mM})$, respectively, the middle cuvette MOAF/DIAF mixed with $5 \mathrm{mM}$ L-Cysteine and the right cuvette MOAF/DIAF commingled with $0.5 \mathrm{mM}$ L-Cysteine hydrochloride monohydrate. The usage of DIAF for Cys detection delivered faster results for the color changes and fluorescence quenching.

The fluorescence measurements of DIAF together with L-Cys hydrochloride monohydrate are shown in Figure 3. A clear correlation between fluorescence emission intensity of DIAF and L-Cys hydrochloride monohydrate concentrations in water ( $\mathrm{pH} 9.5)$ could be observed. The addition of Cys solutions to DIAF lowered its fluorescence emission spectrum within the range from 490 to $540 \mathrm{~nm}$. The lower the Cys concentration the higher signal peaks could be detected.

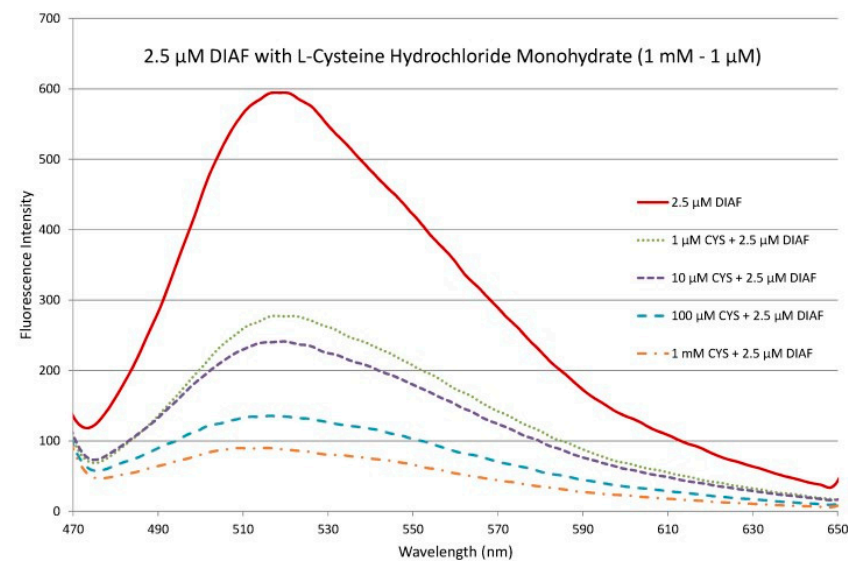

(a)

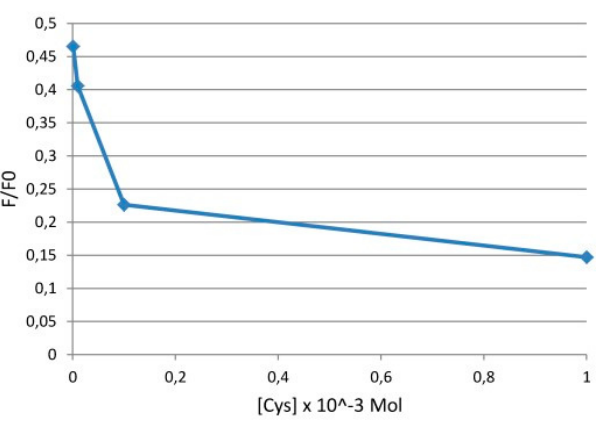

(b)

Figure 3. (a) Fluorescence emission spectra of Fluorescein Dialdehyde $(2.5 \mu \mathrm{M})$ and L-Cysteine hydrochloride monohydrate $(1 \mathrm{mM}, 100 \mu \mathrm{M}, 10 \mu \mathrm{M}$ and $1 \mu \mathrm{M})$ excited at $460 \mathrm{~nm}$ in water, pH 9.5 at room temperature. The fluorescence intensity of DIAF decreases with increasing Cys concentrations. (b) F0 represents the maximum fluorescence intensity in the absence of analyte and F represents the corresponding intensity in the presence of analyte. 
In conclusion Fluorescein Aldehydes proofed to be powerful tools to determine L-Cysteine at very low levels. The presented methodology, especially by using DIAF, shows great promise for fluorescence and UV-vis detection of aminothiols in biological samples. In future experiments we aim to evaluate the cross-reactivity of DIAF solutions with other amino acids, and the exact limits of Cys-detection, and to test its applicability for bio-medical application such as Legionella detection in water samples.

Acknowledgments: The authors thank the Province of Lower Austria and the European Regional Development Fund (EFRE) for their financial support (project ID: WST3-T-91/043-2016).

Conflicts of Interest: The authors declare no conflict of interest. The founding sponsors had no role in the design of the study; in the collection, analyses, or interpretation of data; in the writing of the manuscript, and in the decision to publish the results.

\section{References}

1. Lee, H.; Kim, H.J. Fluorescein aldehyde with disulfide functionality as a fluorescence turn-on probe for cysteine and homocysteine in HEPES buffer. Org. Biomol. Chem. 2013, 11, 5012-5016.

2. Wang, W.; Rusin, O.; Xu, X.; Kim, K.K.; Escobedo, J.O.; Fakayode, S.O.; Fletcher, K.A.; Lowry, M.; Schowalter, C.M.; Lawrence, C.M.; et al. Detection of homocysteine and cysteine J. Am. Chem. Soc. 2005, $127,15949-15958$.

3. Ewann, F.; Hoffman, P.S. Cysteine metabolism in Legionella pneumophilia: Characterization of an LCystine-utilizing mutant. Appl. Environ. Microb. 2006, 72, 3993-4000.

4. Rusin, O.; St. Luce, N.N.; Agbaria, R.A.; Escobedo, J.O.; Jiang, S.; Warner, I.M.; Dawan, F.B.; Lian, K.; Strongin, R.M. Visual Detection of Cysteine and Homocysteine J. Am. Chem. Soc. 2004, 126, 438-439.

5. Burdette, S.C.; Walkup, G.K.; Spingler, B.; Tsien, R.Y.; Lippard, S.J. Fluorescent sensors for Zn2+ based on a fluorescein platform: Synthesis, properties and intracellular distribution J. Am. Chem. Soc. 2001, 123, 78317841.

(C) 2018 by the authors. Licensee MDPI, Basel, Switzerland. This article is an open access article distributed under the terms and conditions of the Creative Commons Attribution (CC BY) license (http://creativecommons.org/licenses/by/4.0/). 